Journal of Microbiological Methods

September 2017, Volume 140 Pages 67-69

http://dx.doi.org/10.1016/i.mimet.2017.07.002

http://archimer.ifremer.fr/doc/00392/50308/

(c) 2017 Elsevier B.V. All rights reserved.

\title{
Development of a duplex Taqman real-time PCR assay for rapid identification of Vibrio splendidus -related and $V$. aestuarianus strains from bacterial cultures
}

\author{
Saulnier Denis ${ }^{1,{ }^{*}}$, De Decker Sophie ${ }^{2}$, Tourbiez Delphine ${ }^{2}$, Travers Marie-Agnes ${ }^{2}$
}

${ }^{1}$ Ifremer, UMR 241 EIO, UPF-ILM-IRD, B.P. 49, 98719 Taravao, Tahiti, French Polynesia

${ }^{2}$ Laboratoire de Génétique et Pathologie des Mollusques Marins, SG2M-RBE Ifremer, av du Mus de Loup, 17390 La Tremblade, French Polynesia

*Corresponding author : Denis Saulnier, email address : denis.saulnier@ifremer.fr

\begin{abstract}
:
To enable the rapid and accurate identification of Vibrio splendidus-related and $V$. aestuarianus strains associated with Pacific cupped oyster Crassostrea gigas mortality, we developed a duplex Taqman realtime PCR assay and evaluated its efficacy. This technique proved to be rapid, sensitive, and specific and will be particularly valuable for epidemiologic studies.
\end{abstract}

Keywords: Multiplex real-time PCR, Gene sequencing, Molluscs, Splendidus clade, Vibrio aestuarianus 
Since 2008, severe seasonal mortality events of juvenile cultured Pacific oyster Crassostrea gigas have been recurrently reported in Europe, especially along the whole French coastline. Mortality rates range from 60 to $90 \%$ (Martenot et al., 2011). Diagnostic analysis of abnormal mortality outbreaks in French mollusc aquaculture, based on the scheme edited by the European Council Directive 2006/88/EC on aquatic animal's health, resulted in the positive detection of one virus, herpes virus type 1 (OsHV-1), and two bacteria, Vibrio splendidus-related and V. aestuarianus, found at a high prevalence of $76 \%$ and $51 \%$, respectively, in 2008 . These infectious agents were shown to exhibit virulent abilities in experimental infection trials (Goudenège et al., 2015; LunaAcosta et al., 2011; Saulnier et al., 2009; Schikorski et al., 2011). Recently, it has been shown that these agents can act solely or in concert (polymicrobial disease) in the field (Lemire et al., 2014; Petton et al., 2015). There is an urgent need to rapidly detect Vibrio splendidus-related and V. aestuarianus to gain a better understanding of the epidemiological significance of their association with or without other infective agents, such as OsHV-1, and the effect of such interactions in detrimental environmental or host factors.

In the past decade, molecular typing methods have revolutionized bacterial species determination 
due to high reproducibility and discriminatory power. They encompass whole-genome typing methods, such as partial or complete genome sequencing, DNA-DNA hybridization, DNA fingerprinting methods and the widely used multilocus sequence typing (MLST) analysis (Sawabe et al., 2007, 2013). Previous studies performed by MLST analysis of housekeeping genes have evidenced that V. splendidus-related strains belong to the Splendidus clade and encompass at least 17 species (Table 2) having a high degree of genetic similarity (Lasa et al., 2013). The recent increase in newly described species isolated from C. gigas oyster (Gonzalez-Castillo et al., 2016, 2014) and our phylogenical analysis on other strains isolated from oyster based on the high discriminatory power of the gyrB gene observed for $V$. splendidus-related strains (Fig. S1) suggest that other Vibrio species not yet described may exist inside this clade. To rapidly identify bacterial colonies obtained by classical culture methods, we developed and validated a Duplex Taqman real time PCR Assay (DTA) for co-determination (in a single reaction tube) of all V. splendidus-related strains (rather than a particular Vibrio species) and V. aestuarianus bacterial isolates, using for the last Vibrio species an already developed real time PCR assay (Saulnier et al., 2009).

Sequence alignment comparisons between housekeeping genes available in Genbank consisted of 16S rRNA, recA, rpoA, toxR and gyrB. We selected one gene, 16S rRNA, which displayed a 25 bp long region that was conserved for all V. splendidus-related strains, except for V. chagasii, V. fortis, V. gallaecicus, V. hemicentroti and V. pelagius, and was divergent from other Vibrio. Taqman probes targeting this region and flanking primers were designed with the aid of Primer 3 software (Table 1). A total of 44 Vibrio species reference strains were used to assess the specificity of the oligonucleotide probes and primers (Table 2). All strains were grown for $96 \mathrm{~h}$ at $20^{\circ} \mathrm{C}$ on plates of Marine agar 2216 (Difco) and DNA extracted by a $10 \mathrm{~min}$ boiling procedure in ultra-pure water (Sigma) as described in Saulnier et al. (2009). Two biological replicates from distinct colonies were 
tested for each bacterial strain. Real-time PCR assay was performed, as described by Saulnier et al. (Saulnier et al., 2009). Briefly, we utilized the same reactive concentrations which included $200 \mathrm{nM}$ of each oligonucleotidic probe and the same PCR thermal profile. Only 14 out of 44 tested Vibrio strains yielded positive amplification signals, with cycle threshold values ranging from 15 to 25 , when selected for either FAM or ROX filters. Moreover, 12 out of 17 strains belonging to the $V$. splendidus-related reference strains were positive using the FAM filter (Splendidus clade probe), whereas five strains belonging to $V$. chagasii, $V$. gallaecicus, $V$. hemicentroti, V. pelagius and $V$. fortis species were negative (Table 2). Only the two $V$. aestuarianus reference strains yielded a positive amplification signal with ROX filter ( $V$. aestuarianus probe). Specificity was determined via agarose gel electrophoresis with 150 and 270-bp long PCR products detected for V. splendidusrelated strains and $V$. aestuarianus, respectively (not shown). All strains were isolated on Marine Agar plate cultures selecting only dominant bacteria by the plate counting method. Of 39 oysters mortality cases analysed in 2008 through active the French surveillance network, 181 bacterial strains were screened using our DTA. A total of 76 strains (42\%) were positive, indicating their affiliation to either $V$. aestuarianus sp. (23 strains) or Splendidus clade (53 strains). To confirm these results using a taxonomic-independent approach, sequencing analysis (Genbank accession numbers in Table S1) was performed on randomly selected strains in each oyster batch (1) from the DTAnegative strains (16SrDNA sequencing on 59 of the 105 negative strains) and (2) from the DTApositive strains (16SrDNA sequencing on 2 of the 23 positive $V$. aestuarianus strains and 8 of the 53 positive Splendidus clade strains and gyrB sequencing on 15 and 24 V. aestuarianus and Splendidus clade-positive strains respectively (Le Roux et al., 2004)). Concerning 16SrDNA sequencing analyses (Fig. S2), results of both tests were in agreement for all DTA-negative and positive $V$. aestuarianus $(67 / 67,2 / 2)$ and nearly all $V$. splendidus-related tested strains (50/61, 8/8), respectively, at the level of $97 \%$ nucleotide identity with $03 / 008^{\mathrm{T}}$ and LMG $4042^{\mathrm{T}}$ reference strains 
(Stackebrandt and Goebel, 1994). However, a mismatch occurred for eleven negative V. splendidusrelated strains that were tentatively classified as $V$. fortis $(\mathrm{n}=4)$ or $V$. chagasii $(\mathrm{n}=7)$. All 15 randomly selected DTA-positive $V$. aestuarianus strains were found to be affiliated to $V$. aestuarianus (15/15) in addition to the 24 arbitrarily selected DTA-positive V. splendidus-related positive strains, as measured by gyrB sequencing, which confirmed the specificity of an already assessed molecular diagnostic test (Saulnier et al., 2009). The specificities of our DTA for $V$. aestuarianus and Spendidus clade were 100\%(67/67) and 100\% (50/50), respectively, and their sensitivities were $100 \%(15 / 15)$ and $82.0 \%(50 / 61)$, respectively. Hence, the use of DTA is a simple and efficient method for rapid (less than two hours), sensitive and specific typing of dominant bacterial strains belonging to either the $V$. aestuarianus $(23 / 181=12.7 \%)$ or the $V$. splendidus group $(53 / 181=29.3 \%)$, both bacteria being closely involved in mortality outbreaks of juvenile Pacific cultured oyster Crassostrea gigas.

This study was supported by the French Ministries of Ecology and Agriculture through the research program of the National Reference Laboratory for mollusc diseases (Conventions DGAL Ifremer). The authors would like to thank the REPAMO national surveillance system for Mollusc Diseases that relies on active institutional surveillance coordinated by the French Food Directorate (DGAL) operating under the European regulatory framework, and who provided the bacterial strains we typed in the present study. We are also grateful to LNR (National Reference Laboratory for mollusc disease) members and particularly Céline Garcia, Maeva Robert, Philippe Haffner and Christine Dubreuil for their participation with bacterial collections.

\section{References}

European Council Directive 2006/88/EC. Official Journal of the European Union, L 328, 24 November 2006. http://eur-lex.europa.eu/legalcontent/EN/TXT/?uri=OJ:L:2006:328:TOCGonzalez-Castillo, A., Balboa, S., Romalde, J.L., Gomez-Gil, B., 2014. Vibrio crosai sp. nov., isolated from a cultured oyster Crassostrea gigas. 
Antonie Van Leeuwenhoek 106, 457-463. doi:10.1007/s10482-014-0214-4

Gonzalez-Castillo, A., Enciso-Ibarra, J., Dubert, J., Romalde, J.L., Gomez-Gil, B., 2016. Vibrio sonorensis sp. nov. isolated from a cultured oyster Crassostrea gigas. Antonie Van Leeuwenhoek 109, 1447-1455. doi:10.1007/s10482-016-0744-z

Goudenège, D., Travers, M.A., Lemire, A., Petton, B., Haffner, P., Labreuche, Y., Tourbiez, D., Mangenot, S., Calteau, A., Mazel, D., Nicolas, J.L., Jacq, A., Le roux, F., 2015. A single regulatory gene is sufficient to alter Vibrio aestuarianus pathogenicity in oysters. Environ. Microbiol. 17, 4189-4199. doi:10.1111/1462-2920.12699

Lasa, A., Dieguez, A.L., Romalde, J.L., 2013. Vibrio toranzoniae sp. nov., a new member of the Splendidus clade in the genus Vibrio. Syst. Appl. Microbiol. 36, 96-100. doi:10.1016/j.syapm.2012.11.005

Le Roux, F., Gay, M., Lambert, C., Nicolas, J.L., Gouy, M., Berthe, F., 2004. Phylogenetic study and identification of Vibrio splendidus-related strains based on gyrB gene sequences. Dis. Aquat. Organ. 58, 143-150. doi:10.3354/dao058143

Lemire, A., Goudenège, D., Versigny, T., Petton, B., Calteau, A., Labreuche, Y., Le Roux, F., 2014. Populations, not clones, are the unit of vibrio pathogenesis in naturally infected oysters. ISME J. December 9, 1-9. doi:10.1038/ismej.2014.233

Luna-Acosta, A., Saulnier, D., Pommier, M., Haffner, P., De Decker, S., Renault, T., ThomasGuyon, H., 2011. First evidence of a potential antibacterial activity involving a laccase-type enzyme of the phenoloxidase system in Pacific oyster Crassostrea gigas haemocytes. Fish Shellfish Immunol. 31, 795-800. doi:10.1016/j.fsi.2011.07.016

Martenot, C., Oden, E., Travaillé, E., Malas, J.-P., Houssin, M., 2011. Detection of different variants of Ostreid Herpesvirus 1 in the Pacific oyster, Crassostrea gigas between 2008 and 2010. Virus Res. doi:10.1016/j.virusres.2011.04.012

Petton, B., Bruto, M., James, A., Labreuche, Y., Alunno-Bruscia, M., Le Roux, F., The Mendeley Support Team, Mendeley Ltd, Dearden, P., Kowalski, B., Lowe, J., Roland, R., Surridge, M., Thomas, S., Jones, S., 2015. Crassostrea gigas mortality in France: The usual suspect, a herpes virus, may not be the killer in this polymicrobial opportunistic disease. Front. Microbiol. 6, 110. doi:10.3389/fmicb.2015.00686

Saulnier, D., De Decker, S., Haffner, P., 2009. Real-time PCR assay for rapid detection and quantification of Vibrio aestuarianus in oyster and seawater: a useful tool for epidemiologic studies. J. Microbiol. Methods 77, 191-7. doi:10.1016/j.mimet.2009.01.021

Sawabe, T., Kita-Tsukamoto, K., Thompson, F.L., 2007. Inferring the evolutionary history of vibrios by means of multilocus sequence analysis. J. Bacteriol. 189, 7932-6. doi:10.1128/JB.00693-07

Sawabe, T., Ogura, Y., Matsumura, Y., Feng, G., Rohul Amin, A.K.M., Mino, S., Nakagawa, S., Sawabe, T., Kumar, R., Fukui, Y., Satomi, M., Matsushima, R., Thompson, F.L., Gomez-Gil, B., Christen, R., Maruyama, F., Kurokawa, K., Hayashi, T., 2013. Updating the Vibrio clades defined by multilocus sequence phylogeny: Proposal of eight new clades, and the description of Vibrio tritonius sp. nov. Front. Microbiol. 4, 1-14. doi:10.3389/fmicb.2013.00414

Schikorski, D., Renault, T., Saulnier, D., Faury, N., Moreau, P., Pépin, J.-F., 2011. Experimental infection of Pacific oyster Crassostrea gigas spat by ostreid herpesvirus 1: Demonstration of oyster spat susceptibility. Vet. Res. 42, 1-27.

Stackebrandt, E., Goebel, B.M., 1994. Taxonomic Note: A Place for DNA-DNA Reassociation and 16S rRNA Sequence Analysis in the Present Species Definition in Bacteriology. Int J Syst Bacteriol 44, 846-849. doi:10.1099/00207713-44-4-846 


\section{Figure captions}

\section{Figure S1 (supplementary data)}

Phylogenetic tree of a partial protein-coding gyrB gene from 41 dominant bacterial strains (all DTA positive) isolated on Zobell Agar plates from Crassotrea gigas oysters sampled in a context of abnormal mortalities (39 episodes along the French coasts in 2008) through the mission of Repamo's French surveillance network. Analysis was performed using Neighbour-Joining method and Kimura 2 parameter model on 487gap-free sites. The evolutionary distances were computed. Bootstrap values (1000 replicates) greater than 50\% are indicated. Genbank accession number of reference type strains is included to identify the strains analysed in this study. DTA-positive Splendidus clade and Vibrio aestuarianus strains are framed by a dashed and solid line respectively.

\section{Figure S2 (supplementary data)}

Phylogenetic tree of partial 16S rDNA from Vibrio isolated from C. gigas oysters collected in the context of mortalities (39 episodes in 2008)and Vibrio reference strains. A total of 591gap-free sites were analysed. The evolutionary distances were computed using the Neighbour-Joining method. Bootstrap values (1000 replicates) greater than 50\% are indicated. Genbank accession number of reference type strains is included to identify the strains analysed in this study. DTA-positive Splendidus clade and Vibrio aestuarianus strains are framed by a dashed and solid line respectively. 


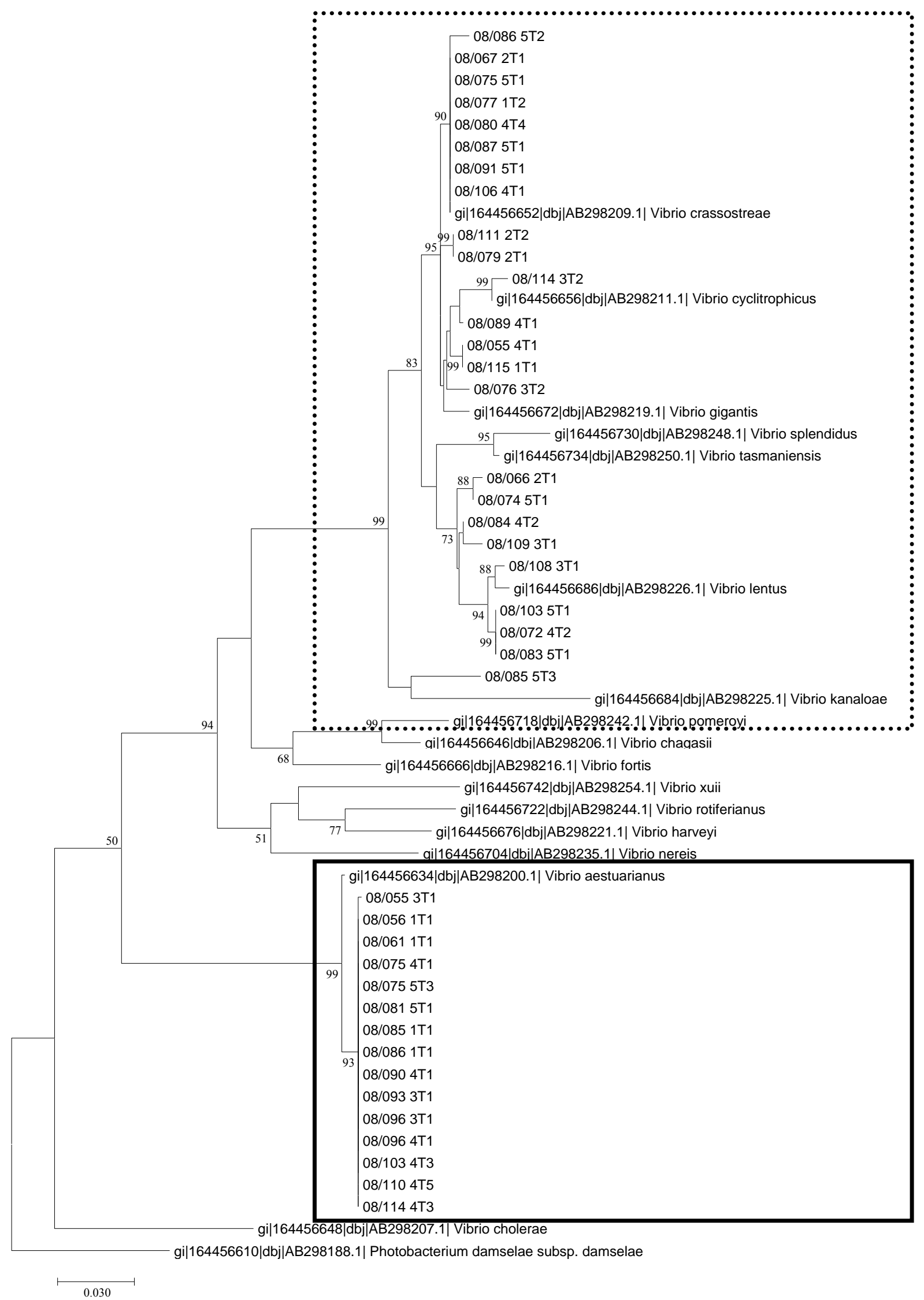

Figure S1 (supplementary data) 


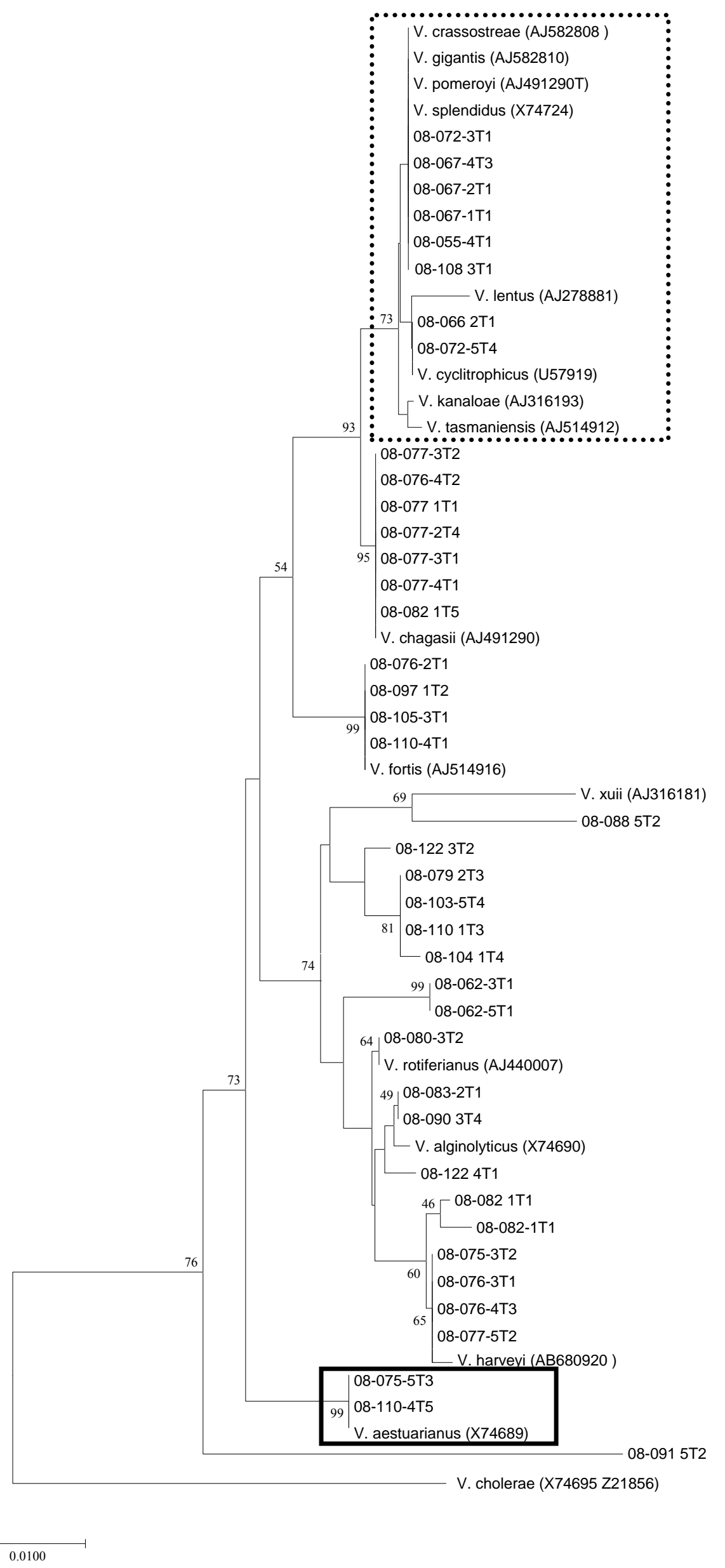

Figure S2 (supplementary data) 


\begin{tabular}{|l|l|l|l|}
\hline Name & Function & Sequence $\left(5^{\prime}-3^{\prime}\right)$ & Tm $\left({ }^{\circ} \mathrm{C}\right)$ \\
\hline $16 \mathrm{~S}$ spF2 & Forward primer & ATCATGGCTCAGATTGAACG & 58 \\
\hline $16 \mathrm{~S}$ spR2 & Reverse primer & CAATGGTTATCCCCCACATC & 60 \\
\hline $16 \mathrm{~S}$ probe & TaqMan PCR probe & FAM-CCCATTAACGCACCCGAAGGATTG BHQ1 & 71 \\
\hline DNAj aes F1 & Forward primer & GTATGAAATTTTAACTGACCCACAA & 58 \\
\hline DNAj aes R1 & Reverse primer & CAATTTCTTTCGAACAACCAC & 59 \\
\hline DNAj probe & TaqMan PCR probe & Texas Red-TGGTAGCGCAGACTTCGGCGAC-BHQ2 & 71 \\
\hline
\end{tabular}

Table 1: Nucleotide sequences and melting temperature $(\mathrm{Tm})$ of primers and Taqman probes for real time PCR reaction. 


\begin{tabular}{|c|c|c|c|}
\hline Clade & Species & Name & DTA results \\
\hline \multirow{3}{*}{ Anguillarum } & Vibrio anguillarum & LMG $4437 \mathrm{~T}$ & - \\
\hline & $\begin{array}{l}\text { Vibrio aestuarianus } \\
\text { subsp. aestuarianus }\end{array}$ & $03 / 008 \mathrm{~T}$ & + \\
\hline & $\begin{array}{l}\text { Vibrio aestuarianus } \\
\text { subsp. francensis }\end{array}$ & $02 / 041 \mathrm{~T}$ & + \\
\hline \multirow{2}{*}{ Coralliilyticus } & Vibrio coralliilyticus & LMG 19607 T & - \\
\hline & Vibrio neptunius & LMG $20536 \mathrm{~T}$ & - \\
\hline \multirow{3}{*}{ Halioticoli } & Vibrio ezurae & DSMZ $17533 \mathrm{~T}$ & - \\
\hline & Vibrio superstes & DSMZ $16383 \mathrm{~T}$ & - \\
\hline & Vibrio gallicus & DSMZ $16639 \mathrm{~T}$ & - \\
\hline \multirow{5}{*}{ Harveyi } & \begin{tabular}{|l} 
Vibrio harveyi \\
\end{tabular} & LMG $4044 \mathrm{~T}$ & - \\
\hline & Vibrio communis & DSMZ $23055 \mathrm{~T}$ & - \\
\hline & V. inhibens & DSMZ $23440 \mathrm{~T}$ & - \\
\hline & Vibrio rotiferianus & DSMZ $17186 \mathrm{~T}$ & - \\
\hline & Vibrio sagamiensis & DSMZ $25651 \mathrm{~T}$ & - \\
\hline \multirow{3}{*}{ Mediterranei } & Vibrio mediterranei & DSMZ $19502 \mathrm{~T}$ & - \\
\hline & Vibrio maritimus & DSMZ $26148 \mathrm{~T}$ & - \\
\hline & Vibrio variabilis & DSMZ $26147 \mathrm{~T}$ & - \\
\hline Nereis & Vibrio xuii & DSMZ $17185 \mathrm{~T}$ & - \\
\hline Nigripulchritudo & Vibrio penaeicida & DSMZ $14398 \mathrm{~T}$ & - \\
\hline orphan & Vibrio tapetis & CECT $4600 \mathrm{~T}$ & - \\
\hline \multirow{5}{*}{ Orientalis } & Vibrio europaeus & $07 / 118 \mathrm{~T} 2$ & - \\
\hline & Vibrio tubiashii & DSMZ $19142 \mathrm{~T}$ & - \\
\hline & Vibrio hepatarius & LMG $20362 \mathrm{~T}$ & - \\
\hline & Vibrio brasiliensis & DSMZ $17184 \mathrm{~T}$ & - \\
\hline & Vibrio atypicus & DSMZ $25292 \mathrm{~T}$ & - \\
\hline \multirow{3}{*}{ not defined } & Vibrio cortegadensis & DSMZ $102805 \mathrm{~T}$ & - \\
\hline & Vibrio shilonii & DSMZ $13774 \mathrm{~T}$ & - \\
\hline & Vibrio pacini & LMG $19999 \mathrm{~T}$ & - \\
\hline \multirow{17}{*}{ Splendidus } & Vibrio artabrorum & DSMZ $26480 \mathrm{~T}$ & + \\
\hline & Vibrio atlanticus & DSMZ $26479 \mathrm{~T}$ & + \\
\hline & Vibrio celticus & DSMZ $26172 \mathrm{~T}$ & + \\
\hline & Vibrio chagasii & LMG $21353 \mathrm{~T}$ & - \\
\hline & Vibrio crassostreae & LGP7 T & + \\
\hline & Vibrio cyclitrophicus & LMG $21359 \mathrm{~T}$ & + \\
\hline & Vibrio fortis & DSMZ $19133 \mathrm{~T}$ & - \\
\hline & Vibrio gallaecicus & DSMZ $23502 \mathrm{~T}$ & - \\
\hline & Vibrio gigantis & LGP13 T & + \\
\hline & Vibrio hemicentroti & DSMZ $26178 \mathrm{~T}$ & - \\
\hline & Vibrio kanaloae & LGM $20539 \mathrm{~T}$ & + \\
\hline & Vibrio lentus & CIP $107166 \mathrm{~T}$ & + \\
\hline & Vibrio pelagius & LMG 3897 T & - \\
\hline & Vibrio pomeroyi & LMG $20537 \mathrm{~T}$ & + \\
\hline & Vibrio splendidus & LMG $4042 \mathrm{~T}$ & + \\
\hline & \begin{tabular}{|l|} 
Vibrio tasmaniensis \\
\end{tabular} & LMG $20012 \mathrm{~T}$ & + \\
\hline & Vibrio toranzoniae & DSMZ $28519 \mathrm{~T}$ & + \\
\hline
\end{tabular}

Table 2: Specificity of the Taqman real time PCR assay according to reference bacterial strains. 


\begin{tabular}{|c|c|c|c|c|c|c|c|}
\hline $\begin{array}{c}\text { Strain } \\
\text { number }\end{array}$ & $\begin{array}{c}\text { Mortality } \\
\text { batches }\end{array}$ & $\begin{array}{l}\text { Sampling } \\
\text { period }\end{array}$ & $\begin{array}{c}\text { Origin and } \\
\text { (department } \\
\text { number) }\end{array}$ & $\begin{array}{c}\text { Oyster } \\
\text { age } \\
\text { class }\end{array}$ & $\begin{array}{c}\text { DTA } \\
\text { identification }\end{array}$ & $\begin{array}{c}\text { 16s rRNA } \\
\text { GenBank } \\
\text { number }\end{array}$ & $\begin{array}{c}\text { gyrB } \\
\text { GenBank } \\
\text { number }\end{array}$ \\
\hline $\begin{array}{c}08-055 \\
3 \mathrm{~T} 1\end{array}$ & 08-055 & $15 / 05 / 2008$ & $\begin{array}{l}\text { La Flotte en } \\
\text { Ré (17) }\end{array}$ & spat & V. aestuarianus & & KY930534 \\
\hline $\begin{array}{c}08-056 \\
1 \mathrm{~T} 1\end{array}$ & 08-056 & $15 / 05 / 2008$ & $\begin{array}{l}\text { La Flotte en } \\
\text { Ré (17) }\end{array}$ & adult & $V$. aestuarianus & & KY930535 \\
\hline $\begin{array}{c}08-061 \\
1 \mathrm{~T} 1\end{array}$ & 08-061 & $20 / 05 / 2008$ & Thau (34) & juvenile & $V$. aestuarianus & & KY930536 \\
\hline $\begin{array}{c}08-075 \\
4 \mathrm{~T} 1\end{array}$ & $08-075$ & $30 / 06 / 2008$ & Arcachon (33) & spat & V. aestuarianus & & KY930537 \\
\hline $\begin{array}{l}08-075 \\
5 \mathrm{~T} 3\end{array}$ & 08-075 & $30 / 06 / 2008$ & Arcachon (33) & spat & V. aestuarianus & KY923251 & KY930538 \\
\hline $\begin{array}{c}08-081 \\
5 \mathrm{~T} 1\end{array}$ & 08-081 & $30 / 06 / 2008$ & Ronce-Perquis & juvenile & $V$. aestuarianus & & KY930539 \\
\hline $\begin{array}{c}08-085 \\
1 \mathrm{~T} 1 \\
\end{array}$ & 08-085 & 03/07/2008 & $\begin{array}{l}\text { Penthièvre } \\
\quad(56)\end{array}$ & spat & V. aestuarianus & & KY930540 \\
\hline $\begin{array}{c}08-086 \\
1 \mathrm{~T} 1\end{array}$ & 08-086 & 03/07/2008 & $\begin{array}{c}\text { Penthièvre } \\
(56)\end{array}$ & spat & $V$. aestuarianus & & KY930541 \\
\hline $\begin{array}{c}08-090 \\
4 \mathrm{~T} 1\end{array}$ & 08-090 & 04/07/2008 & $\begin{array}{l}\text { Chatelaillon } \\
\text { (17) }\end{array}$ & juvenile & V. aestuarianus & & KY930542 \\
\hline $\begin{array}{c}08-093 \\
3 \mathrm{~T} 1 \\
\end{array}$ & 08-093 & 07/07/2008 & $\begin{array}{l}\text { Aiguillon sur } \\
\text { Mer (17) }\end{array}$ & juvenile & V. aestuarianus & & KY930543 \\
\hline $\begin{array}{c}08-096 \\
3 \mathrm{~T} 1\end{array}$ & 08-096 & 09/07/2008 & $\begin{array}{c}\text { Rivière } \\
\text { d'Auray (56) }\end{array}$ & $\begin{array}{c}\text { spat/ } \\
\text { juvenile }\end{array}$ & V. aestuarianus & & KY930544 \\
\hline $\begin{array}{c}08-096 \\
4 \mathrm{~T} 1\end{array}$ & 08-096 & 09/07/2008 & $\begin{array}{c}\text { Rivière } \\
\text { d'Auray (56) }\end{array}$ & $\begin{array}{c}\text { spat/ } \\
\text { juvenile }\end{array}$ & V. aestuarianus & & KY930545 \\
\hline $\begin{array}{c}08 / 103 \\
4 \mathrm{~T} 3\end{array}$ & 08/103 & $17 / 07 / 2008$ & $\begin{array}{c}\text { Rivière } \\
\text { d'Auray (56) }\end{array}$ & adult & V. aestuarianus & & KY930546 \\
\hline $\begin{array}{l}08 / 110 \\
4 \mathrm{~T} 5\end{array}$ & 08/110 & $20 / 07 / 2008$ & $\begin{array}{l}\text { Ronce les } \\
\text { bains (17) }\end{array}$ & adults & V. aestuarianus & KY923252 & KY930547 \\
\hline $\begin{array}{c}08 / 114 \\
4 \mathrm{~T} 3\end{array}$ & 08/114 & $21 / 07 / 2008$ & $\begin{array}{l}\text { Saint Germain } \\
\text { sur Ay (50) }\end{array}$ & juvenile & V. aestuarianus & & KY930548 \\
\hline $\begin{array}{c}08-055 \\
4 \mathrm{~T} 1\end{array}$ & 08-055 & $15 / 05 / 2008$ & $\begin{array}{l}\text { La Flotte en } \\
\text { Ré (17) }\end{array}$ & spat & $\begin{array}{l}V . \text { splendidus- } \\
\text { related }\end{array}$ & KY923253 & KY930549 \\
\hline $\begin{array}{c}08-066 \\
2 \mathrm{~T} 1\end{array}$ & 08-066 & $28 / 05 / 2008$ & $\begin{array}{c}\text { Golfe du } \\
\text { Morbihan (56) }\end{array}$ & spat & $\begin{array}{l}V . \text { splendidus- } \\
\text { related }\end{array}$ & KY923254 & KY930550 \\
\hline $\begin{array}{c}08-067 \\
1 \mathrm{~T} 1\end{array}$ & 08-067 & $05 / 06 / 2008$ & $\begin{array}{c}\text { Esnandes- } \\
\text { Marsilly (17) }\end{array}$ & spat & $\begin{array}{l}V . \text { splendidus- } \\
\text { related }\end{array}$ & KY923255 & \\
\hline $\begin{array}{c}08-067 \\
2 \mathrm{~T} 1\end{array}$ & 08-067 & 05/06/2008 & $\begin{array}{c}\text { Esnandes- } \\
\text { Marsilly (17) }\end{array}$ & spat & $\begin{array}{l}V . \text { splendidus- } \\
\text { related }\end{array}$ & KY923256 & KY930551 \\
\hline $\begin{array}{c}08-067 \\
4 \mathrm{~T} 3\end{array}$ & 08-067 & $05 / 06 / 2008$ & $\begin{array}{c}\text { Esnandes- } \\
\text { Marsilly (17) }\end{array}$ & spat & $\begin{array}{l}V . \text { splendidus- } \\
\text { related }\end{array}$ & KY923257 & \\
\hline $\begin{array}{c}08-072 \\
3 \mathrm{~T} 1 \\
\end{array}$ & 08-072 & $19 / 06 / 2008$ & $\begin{array}{c}\text { Baie des Veys } \\
(50)\end{array}$ & juvenile & $\begin{array}{l}V . \text { splendidus- } \\
\text { related }\end{array}$ & KY923258 & \\
\hline $\begin{array}{c}08-072 \\
4 \mathrm{~T} 2\end{array}$ & 08-072 & $19 / 06 / 2008$ & $\begin{array}{l}\text { Baie des Veys } \\
\qquad(50)\end{array}$ & juvenile & $\begin{array}{l}V . \text { splendidus- } \\
\text { related }\end{array}$ & & KY930552 \\
\hline
\end{tabular}




\begin{tabular}{|c|c|c|c|c|c|c|c|}
\hline $\begin{array}{c}08-072 \\
5 \mathrm{~T} 4\end{array}$ & $08-072$ & $19 / 06 / 2008$ & $\begin{array}{c}\text { Baie des Veys } \\
(50)\end{array}$ & juvenile & $\begin{array}{l}\text { V. splendidus- } \\
\text { related }\end{array}$ & KY923259 & \\
\hline $\begin{array}{c}08-074 \\
5 \mathrm{~T} 1\end{array}$ & $08-074$ & 01/07/2008 & Meuvaine (50) & juvenile & $\begin{array}{l}V . \text { splendidus- } \\
\text { related }\end{array}$ & & KY930553 \\
\hline $\begin{array}{c}08-075 \\
5 \mathrm{~T} 1\end{array}$ & $08-075$ & $30 / 06 / 2008$ & Arcachon (33) & spat & $\begin{array}{l}V . \text { splendidus- } \\
\text { related }\end{array}$ & & KY930554 \\
\hline $\begin{array}{c}08-076 \\
3 \mathrm{~T} 2\end{array}$ & $08-076$ & $02 / 07 / 2008$ & $\begin{array}{l}\text { Marseillan } \\
\text { (34) }\end{array}$ & spat & $\begin{array}{l}\text { V. splendidus- } \\
\text { related }\end{array}$ & & KY930555 \\
\hline $\begin{array}{c}08-077 \\
1 \mathrm{~T} 2\end{array}$ & 08-077 & 02/07/2008 & Thau (34) & spat & $\begin{array}{l}V . \text { splendidus- } \\
\text { related }\end{array}$ & & KY930556 \\
\hline $\begin{array}{c}08-079 \\
2 \mathrm{~T} 1\end{array}$ & 08-079 & $02 / 07 / 2008$ & Blainville (50) & spat & $\begin{array}{l}\text { V. splendidus- } \\
\text { related }\end{array}$ & & KY930557 \\
\hline $\begin{array}{c}08-080 \\
4 \mathrm{~T} 4\end{array}$ & 08-080 & $30 / 06 / 2008$ & $\begin{array}{c}\text { Ronce-Perquis } \\
\text { (17) }\end{array}$ & spat & $\begin{array}{l}V . \text { splendidus- } \\
\text { related }\end{array}$ & & KY930558 \\
\hline $\begin{array}{c}08-083 \\
5 \mathrm{~T} 1\end{array}$ & $08-083$ & $02 / 07 / 2008$ & Blainville (50) & juvenile & $\begin{array}{l}\text { V. splendidus- } \\
\text { related }\end{array}$ & & KY930559 \\
\hline $\begin{array}{c}08-084 \\
4 \mathrm{~T} 2\end{array}$ & 08-084 & $02 / 07 / 2008$ & Gouville (50) & spat & $\begin{array}{l}\text { V. splendidus- } \\
\text { related }\end{array}$ & & KY930560 \\
\hline $\begin{array}{c}08-085 \\
5 \mathrm{~T} 3\end{array}$ & 08-085 & 03/07/2008 & $\begin{array}{l}\text { Penthièvre } \\
\text { (56) }\end{array}$ & spat & $\begin{array}{l}\text { V. splendidus- } \\
\text { related }\end{array}$ & & KY930561 \\
\hline $\begin{array}{c}08-086 \\
5 \mathrm{~T} 2\end{array}$ & 08-086 & 03/07/2008 & $\begin{array}{l}\text { Penthièvre } \\
\text { (56) }\end{array}$ & spat & $\begin{array}{l}\text { V. splendidus- } \\
\text { related }\end{array}$ & & KY930562 \\
\hline $\begin{array}{c}08-087 \\
5 \mathrm{~T} 1\end{array}$ & 08-087 & 03/07/2008 & Etel (56) & spat & $\begin{array}{l}\text { V. splendidus- } \\
\text { related }\end{array}$ & & KY930563 \\
\hline $\begin{array}{c}08-089 \\
4 \mathrm{~T} 1 \\
\end{array}$ & 08-089 & $04 / 07 / 2008$ & $\begin{array}{c}\text { Bretagne Nord } \\
(29)\end{array}$ & spat & $\begin{array}{l}\text { V. splendidus- } \\
\text { related }\end{array}$ & & KY930564 \\
\hline $\begin{array}{c}08-091 \\
5 \mathrm{~T} 1 \\
\end{array}$ & 08-091 & 07/07/2008 & Cancale (35) & juvenile & $\begin{array}{l}\text { V. splendidus- } \\
\text { related }\end{array}$ & & KY930565 \\
\hline $\begin{array}{c}08 / 103 \\
5 \mathrm{~T} 1\end{array}$ & $08 / 103$ & $17 / 07 / 2008$ & $\begin{array}{c}\text { Rivière } \\
\text { dAuray (56) }\end{array}$ & adult & $\begin{array}{l}\text { V. splendidus- } \\
\text { related }\end{array}$ & & KY930566 \\
\hline $\begin{array}{c}08 / 106 \\
4 \mathrm{~T} 1\end{array}$ & $08 / 106$ & $17 / 07 / 2008$ & $\begin{array}{c}\text { Bonne anse } \\
\text { (17) }\end{array}$ & spat & $\begin{array}{l}\text { V. splendidus- } \\
\text { related }\end{array}$ & & KY930567 \\
\hline $\begin{array}{c}08 / 108 \\
3 \mathrm{~T} 1 \\
\end{array}$ & $08 / 108$ & $21 / 07 / 2008$ & $\begin{array}{c}\text { Baie des Veys } \\
(50)\end{array}$ & spat & $\begin{array}{l}\text { V. splendidus- } \\
\text { related }\end{array}$ & KY923260 & KY930568 \\
\hline $\begin{array}{c}08 / 109 \\
3 \mathrm{~T} 1\end{array}$ & 08/109 & $21 / 07 / 2008$ & $\begin{array}{c}\text { Baie des Veys } \\
(50)\end{array}$ & juvenile & $\begin{array}{l}\text { V. splendidus- } \\
\text { related }\end{array}$ & & KY930569 \\
\hline $\begin{array}{l}08 / 111 \\
2 \mathrm{~T} 2\end{array}$ & $08 / 111$ & $21 / 07 / 2008$ & Ile d'aix (17) & adult & $\begin{array}{l}\text { V. splendidus- } \\
\text { related }\end{array}$ & & KY930570 \\
\hline $\begin{array}{c}08 / 114 \\
3 \mathrm{~T} 2\end{array}$ & $08 / 114$ & $21 / 07 / 2008$ & $\begin{array}{c}\text { Saint Germain } \\
\text { sur Ay (50) }\end{array}$ & juvenile & $\begin{array}{l}\text { V. splendidus- } \\
\text { related }\end{array}$ & & KY930571 \\
\hline $\begin{array}{c}08 / 115 \\
1 \mathrm{~T} 1 \\
\end{array}$ & $08 / 115$ & $22 / 07 / 2008$ & $\begin{array}{c}\text { Etang prevost } \\
(80)\end{array}$ & spat & $\begin{array}{l}\text { V. splendidus- } \\
\text { related }\end{array}$ & & KY930572 \\
\hline $\begin{array}{c}08-062 \\
3 \mathrm{~T} 1\end{array}$ & $08-062$ & $23 / 05 / 2008$ & Ars en Ré (17) & juvenile & negative & KY923649 & \\
\hline $\begin{array}{c}08-062 \\
5 \mathrm{~T} 1\end{array}$ & $08-062$ & $23 / 05 / 2008$ & Ars en Ré (17) & juvenile & negative & KY923650 & \\
\hline $\begin{array}{c}08-066 \\
5 \mathrm{~T} 2\end{array}$ & $08-066$ & $28 / 05 / 2008$ & $\begin{array}{c}\text { Golfe du } \\
\text { Morbihan (56) }\end{array}$ & spat & negative & KY923651 & \\
\hline $\begin{array}{c}08-067 \\
2 \mathrm{~T} 2\end{array}$ & $08-067$ & $05 / 06 / 2008$ & $\begin{array}{l}\text { Esnandes- } \\
\text { Marsilly (17) }\end{array}$ & spat & negative & KY923652 & \\
\hline $\begin{array}{c}08-067 \\
3 \mathrm{~T} 3\end{array}$ & $08-067$ & $05 / 06 / 2008$ & $\begin{array}{c}\text { Esnandes- } \\
\text { Marsilly (17) }\end{array}$ & spat & negative & KY923653 & \\
\hline $\begin{array}{c}08-072 \\
4 \mathrm{~T} 1 \\
\end{array}$ & 08-072 & $19 / 06 / 2008$ & $\begin{array}{c}\text { Baie des Veys } \\
(50)\end{array}$ & juvenile & negative & KY923654 & \\
\hline
\end{tabular}




\begin{tabular}{|c|c|c|c|c|c|c|}
\hline $\begin{array}{c}08-072 \\
4 \mathrm{~T} 5\end{array}$ & 08-072 & $19 / 06 / 2008$ & $\begin{array}{l}\text { Baie des Veys } \\
\quad(50)\end{array}$ & juvenile & negative & KY923655 \\
\hline $\begin{array}{c}\text { 08-072 } \\
\text { 4T6 }\end{array}$ & 08-072 & $19 / 06 / 2008$ & $\begin{array}{l}\text { Baie des Veys } \\
(50)\end{array}$ & juvenile & negative & KY923656 \\
\hline $\begin{array}{c}08-074 \\
4 \mathrm{~T} 1\end{array}$ & $08-074$ & $01 / 07 / 2008$ & Meuvaine (50) & juvenile & negative & KY923657 \\
\hline $\begin{array}{c}08-075 \\
3 \mathrm{~T} 2\end{array}$ & $08-075$ & $30 / 06 / 2008$ & Arcachon (33) & spat & negative & KY923659 \\
\hline $\begin{array}{c}08-075 \\
3 \mathrm{~T} 3 \\
\end{array}$ & $08-075$ & $30 / 06 / 2008$ & Arcachon (33) & spat & negative & KY923658 \\
\hline $\begin{array}{c}08-076 \\
2 \mathrm{~T} 1 \\
\end{array}$ & $08-076$ & 02/07/2008 & $\begin{array}{l}\text { Marseillan } \\
\text { (34) }\end{array}$ & spat & negative & KY923261 \\
\hline $\begin{array}{c}\text { 08-076 } \\
3 \mathrm{~T} 1\end{array}$ & 08-076 & $02 / 07 / 2008$ & $\begin{array}{l}\text { Marseillan } \\
\text { (34) }\end{array}$ & spat & negative & KY923660 \\
\hline $\begin{array}{c}\text { 08-076 } \\
\text { 4T2 }\end{array}$ & $08-076$ & 02/07/2008 & $\begin{array}{l}\text { Marseillan } \\
(34) \\
\end{array}$ & spat & negative & KY923262 \\
\hline $\begin{array}{c}08-076 \\
4 \mathrm{~T} 3\end{array}$ & $08-076$ & 02/07/2008 & $\begin{array}{l}\text { Marseillan } \\
(34)\end{array}$ & spat & negative & KY923661 \\
\hline $\begin{array}{c}\text { 08-077 } \\
1 \mathrm{~T} 1\end{array}$ & 08-077 & $02 / 07 / 2008$ & Thau (34) & spat & negative & KY923263 \\
\hline $\begin{array}{c}08-077 \\
2 \mathrm{~T} 4\end{array}$ & 08-077 & $02 / 07 / 2008$ & Thau (34) & spat & negative & KY923264 \\
\hline $\begin{array}{c}08-077 \\
3 \mathrm{~T} 1\end{array}$ & 08-077 & $02 / 07 / 2008$ & Thau (34) & spat & negative & KY923265 \\
\hline $\begin{array}{c}08-077 \\
4 \mathrm{~T} 1\end{array}$ & 08-077 & 02/07/2008 & Thau (34) & spat & negative & KY923266 \\
\hline $\begin{array}{c}\text { 08-077 } \\
4 \mathrm{~T} 2\end{array}$ & 08-077 & $02 / 07 / 2008$ & Thau (34) & spat & negative & KY923662 \\
\hline $\begin{array}{c}08-077 \\
5 \mathrm{~T} 2\end{array}$ & 08-077 & 02/07/2008 & Thau (34) & spat & negative & KY923663 \\
\hline $\begin{array}{c}08-079 \\
2 \mathrm{~T} 3\end{array}$ & 08-079 & 02/07/2008 & Blainville (50) & spat & negative & KY923664 \\
\hline $\begin{array}{c}08-080 \\
3 \mathrm{~T} 2\end{array}$ & 08-080 & $30 / 06 / 2008$ & $\begin{array}{l}\text { Ronce-Perquis } \\
\text { (17) }\end{array}$ & spat & negative & KY923665 \\
\hline $\begin{array}{c}08-080 \\
4 \mathrm{~T} 1\end{array}$ & 08-080 & $30 / 06 / 2008$ & $\begin{array}{c}\text { Ronce-Perquis } \\
(17)\end{array}$ & spat & negative & KY923666 \\
\hline $\begin{array}{c}\text { 08-081 } \\
\text { 4T1 }\end{array}$ & 08-081 & $30 / 06 / 2008$ & $\begin{array}{c}\text { Ronce-Perquis } \\
\text { (17) }\end{array}$ & juvenile & negative & KY923667 \\
\hline $\begin{array}{c}08-081 \\
5 \mathrm{~T} 2\end{array}$ & 08-081 & $30 / 06 / 2008$ & $\begin{array}{c}\text { Ronce-Perquis } \\
\text { (17) }\end{array}$ & juvenile & negative & KY923668 \\
\hline $\begin{array}{c}\text { 08-081 } \\
5 \mathrm{~T} 3\end{array}$ & 08-081 & $30 / 06 / 2008$ & $\begin{array}{c}\text { Ronce-Perquis } \\
\text { (17) }\end{array}$ & juvenile & negative & KY923669 \\
\hline $\begin{array}{c}\text { 08-082 } \\
\text { 1T1 }\end{array}$ & 08-082 & $02 / 07 / 2008$ & $\begin{array}{c}\text { Baie de } \\
\text { Bourgneuf } \\
(44)\end{array}$ & larvae & negative & KY923267 \\
\hline $\begin{array}{c}08-082 \\
1 \mathrm{~T} 2\end{array}$ & 08-082 & $02 / 07 / 2008$ & $\begin{array}{l}\text { Baie de } \\
\text { Bourgneuf } \\
(44)\end{array}$ & larvae & negative & KY923670 \\
\hline $\begin{array}{c}08-082 \\
1 \mathrm{~T} 5\end{array}$ & 08-082 & $02 / 07 / 2008$ & $\begin{array}{l}\text { Baie de } \\
\text { Bourgneuf } \\
(44)\end{array}$ & larvae & negative & KY923268 \\
\hline $\begin{array}{c}\text { 08-083 } \\
2 \mathrm{~T} 1\end{array}$ & $08-083$ & $02 / 07 / 2008$ & Blainville (50) & juvenile & negative & KY923671 \\
\hline $\begin{array}{c}08-084 \\
3 \mathrm{~T} 2\end{array}$ & 08-084 & $02 / 07 / 2008$ & Gouville (50) & spat & negative & KY923672 \\
\hline $\begin{array}{c}08-084 \\
4 \mathrm{~T} 1\end{array}$ & 08-084 & $02 / 07 / 2008$ & Gouville (50) & spat & negative & KY923673 \\
\hline $\begin{array}{c}08-085 \\
5 \mathrm{~T} 2\end{array}$ & 08-085 & 03/07/2008 & $\begin{array}{l}\text { Penthièvre } \\
\text { (56) }\end{array}$ & spat & negative & KY923674 \\
\hline
\end{tabular}




\begin{tabular}{|c|c|c|c|c|c|c|}
\hline $\begin{array}{c}08-087 \\
5 \mathrm{~T} 2\end{array}$ & 08-087 & 03/07/2008 & Etel (56) & spat & negative & KY923675 \\
\hline $\begin{array}{c}08-088 \\
5 \mathrm{~T} 2 \\
\end{array}$ & 08-088 & $03 / 07 / 2008$ & Etel (56) & spat & negative & KY923676 \\
\hline $\begin{array}{c}08-090 \\
3 \mathrm{~T} 4\end{array}$ & 08-090 & $04 / 07 / 2008$ & $\begin{array}{c}\text { Chatelaillon } \\
\text { (17) }\end{array}$ & juvenile & negative & KY923677 \\
\hline $\begin{array}{c}08-091 \\
3 \mathrm{~T} 1\end{array}$ & 08-091 & 07/07/2008 & Cancale (35) & juvenile & negative & KY923678 \\
\hline $\begin{array}{c}\text { 08-091 } \\
5 \mathrm{~T} 2\end{array}$ & 08-091 & $07 / 07 / 2008$ & Cancale (35) & juvenile & negative & KY923679 \\
\hline $\begin{array}{c}08-097 \\
1 \mathrm{~T} 1\end{array}$ & 08-097 & 08/07/2008 & $\begin{array}{c}\text { Baie de } \\
\text { Bourgneuf } \\
(44)\end{array}$ & juvenile & negative & KY923680 \\
\hline $\begin{array}{c}08-097 \\
1 \mathrm{~T} 2\end{array}$ & 08-097 & $08 / 07 / 2008$ & $\begin{array}{c}\text { Baie de } \\
\text { Bourgneuf } \\
(44)\end{array}$ & juvenile & negative & KY923269 \\
\hline $\begin{array}{c}08 / 103 \\
3 \mathrm{~T} 2 \\
\end{array}$ & 08/103 & $17 / 07 / 2008$ & $\begin{array}{c}\text { Rivière } \\
\text { dAuray (56) }\end{array}$ & adult & negative & KY923681 \\
\hline $\begin{array}{c}08 / 103 \\
5 \mathrm{~T} 4\end{array}$ & 08/103 & $17 / 07 / 2008$ & $\begin{array}{c}\text { Rivière } \\
\text { dAuray (56) }\end{array}$ & adult & negative & KY923682 \\
\hline $\begin{array}{c}08 / 104 \\
1 \mathrm{~T} 4 \\
\end{array}$ & 08/104 & $17 / 07 / 2008$ & $\begin{array}{l}\text { Ronce les } \\
\text { bains (17) }\end{array}$ & larvae & negative & KY923683 \\
\hline $\begin{array}{c}08 / 105 \\
3 \mathrm{~T} 1\end{array}$ & $08 / 105$ & $17 / 07 / 2008$ & $\begin{array}{l}\text { Ronce les } \\
\text { bains (17) }\end{array}$ & adult & negative & KY923270 \\
\hline $\begin{array}{c}08 / 106 \\
3 \mathrm{~T} 2\end{array}$ & 08/106 & $17 / 07 / 2008$ & $\begin{array}{c}\text { Bonne anse } \\
\text { (17) }\end{array}$ & spat & negative & KY923684 \\
\hline $\begin{array}{c}08 / 108 \\
4 \mathrm{~T} 1\end{array}$ & 08/108 & $21 / 07 / 2008$ & $\begin{array}{c}\text { Baie des Veys } \\
(50)\end{array}$ & spat & negative & KY923685 \\
\hline $\begin{array}{c}08 / 110 \\
1 \mathrm{~T} 2\end{array}$ & $08 / 110$ & $20 / 07 / 2008$ & $\begin{array}{l}\text { Ronce les } \\
\text { bains (17) }\end{array}$ & adult & negative & KY923686 \\
\hline $\begin{array}{c}08 / 110 \\
1 \mathrm{~T} 3\end{array}$ & $08 / 110$ & $20 / 07 / 2008$ & $\begin{array}{l}\text { Ronce les } \\
\text { bains (17) }\end{array}$ & adult & negative & KY923687 \\
\hline $\begin{array}{c}08 / 110 \\
3 \mathrm{~T} 1\end{array}$ & $08 / 110$ & $20 / 07 / 2008$ & $\begin{array}{l}\text { Ronce les } \\
\text { bains (17) }\end{array}$ & adult & negative & KY923688 \\
\hline $\begin{array}{c}08 / 110 \\
4 \mathrm{~T} 1 \\
\end{array}$ & $08 / 110$ & $20 / 07 / 2008$ & $\begin{array}{l}\text { Ronce les } \\
\text { bains (17) }\end{array}$ & adult & negative & KY923271 \\
\hline $\begin{array}{c}08 / 111 \\
1 \mathrm{~T} 1 \\
\end{array}$ & $08 / 111$ & $21 / 07 / 2008$ & Ile d'aix (17) & adult & negative & KY923689 \\
\hline $\begin{array}{c}08 / 111 \\
2 \mathrm{~T} 1 \\
\end{array}$ & $08 / 111$ & $21 / 07 / 2008$ & Ile d'aix (17) & adult & negative & KY923690 \\
\hline $\begin{array}{c}08 / 111 \\
3 \mathrm{~T} 1\end{array}$ & $08 / 111$ & $21 / 07 / 2008$ & Ile d'aix (17) & adult & negative & KY923691 \\
\hline 08/114 1t1 & $08 / 114$ & $21 / 07 / 2008$ & $\begin{array}{c}\text { Saint Germain } \\
\text { sur Ay (50) }\end{array}$ & juvenile & negative & KY923692 \\
\hline $\begin{array}{c}08 / 114 \\
4 \mathrm{~T} 2\end{array}$ & $08 / 114$ & $21 / 07 / 2008$ & $\begin{array}{l}\text { Saint Germain } \\
\text { sur Ay (50) }\end{array}$ & juvenile & negative & KY923693 \\
\hline $\begin{array}{c}08 / 114 \\
5 \mathrm{~T} 1\end{array}$ & $08 / 114$ & $21 / 07 / 2008$ & $\begin{array}{c}\text { Saint Germain } \\
\text { sur Ay (50) }\end{array}$ & juvenile & negative & KY923694 \\
\hline $\begin{array}{c}08 / 122 \\
3 \mathrm{~T} 2\end{array}$ & $08 / 122$ & $07 / 08 / 2008$ & Arcachon (33) & spat & negative & KY923695 \\
\hline $\begin{array}{c}08 / 122 \\
4 \mathrm{~T} 1\end{array}$ & $08 / 122$ & $07 / 08 / 2008$ & Arcachon (33) & spat & negative & KY923696 \\
\hline
\end{tabular}

Table S1 (supplementary data): Genbank accession numbers and origin of the bacterial strains isolated from Crassostrea gigas in 2008. Their sources (sampling period, age class of the oysters and origin of the mortality batches), as well as DTA results and Genbank accession numbers (for 16s rDNA and gyrB genes) are indicated. 